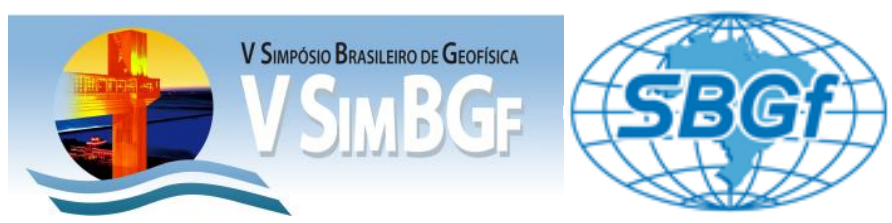

\title{
Um breve estudo de uma tempestade magnética e a formação de bolhas de plasma na região Amazônica, sobre Manaus (Lat. -3,10 e Long. -59,97).
}

\author{
Verônica Ingrid Zuim Rego ${ }^{1}$,Newton Silva de Lima ${ }^{1}$, Alan dos Santos Ferreira1, Kaio Barbosa1, Matheus da Rocha Pietzsch ${ }^{1}$ \\ (1) Centro Universitário Luterano de Manaus \\ Av. Carlos Drummond de Andrade, 1460 - Cj. Atíllio Andreazza - JAPIIM 2 \\ 69077-730 - Manaus, AM - Brasil \\ veronicazure@hotmail.com; newtonulbra@gmail.com
}

Copyright 2012, SBGf - Sociedade Brasileira de Geofísica

Este texto foi preparado para a apresentação no V Simpósio Brasileiro de Geofísica, Salvador, 27a 29 de novembro de 2012. Seu conteúdo foi revisado pelo Comitê Técnico do VSimBGt mas não necessariamente representa a opinião da SBGf ou de seus associados. É proibida a reprodução total ou parcial deste material para propósitos comerciais sem prévia autorização da SBGf.

\section{Resumo}

Este artigo apresenta o resultado do estudo realizado com dados do conteúdo total eletrônico (TEC). Para isto foi utilizado o sistema de posicionamento global (GPSNovAtel), da rede de sensores ionosféricos na região equatorial Amazônica. O mês estudado é o de março 2012. O objetivo principal é investigar as anomalias ionosféricas equatoriais de grande escala na variação diária do TEC e identificação de tempestade magnética através do espectro de potência de ondeleta.

\section{Introdução}

O Sol emite radiação ao longo de todo espectro eletromagnético, desde radiação gama até as ondas de rádio, passando pela radiação- $x$, ultravioleta, visível, infravermelho, microondas. A maior parte da intensidade concentra-se na faixa do visível. (ECSS, 2000).

De acordo Lima, (2009), a Amazônia está situada em uma região de máximo na distribuição global de ocorrência das bolhas de plasma (irregularidade ionosféricas de grande escala), ou seja, no Brasil ocorrem mais bolhas que em qualquer outra região do planeta (BURKE et al., 2004). As irregularidades de grande escala mencionadas são regiões onde a densidade do plasma é menor que a densidade do plasma ambiente e são alinhadas ao meridiano magnético. Estas irregularidades são geradas na região equatorial, logo após o entardecer e se propagam normalmente para leste até se dissiparem. É amplamente aceito que estas irregularidades são geradas pela instabilidade do tipo Rayleigh-Taylor.

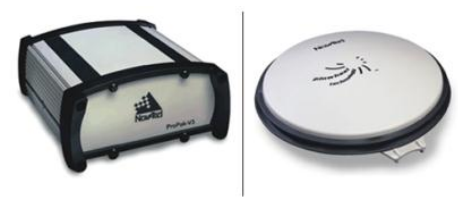

Figura 1. Receptor e antena (GPS - NovAtel - NOV GSV4004B - Antena NOV702rev.4) (Fonte:UNIVAP-SJC$\mathrm{SP})$

\section{Metodologia/ Problema Investigado}

A pesquisa tem como base o monitoramento do ciclo solar \# 24, na região equatorial, através de um GPS ionosférico (NovAtel - NOV GSV4004B - Antena NOV702rev.4), Fig. 1, Tab. 1. Esta é realizada com o auxílio da análise gráficas do TEC (Total Electron Content) (Fig. 2), os quais mostram a variação da intensidade de elétrons livres por área na ionosfera terrestre. A quantidade de elétrons ao longo do comprimento do percurso é referida como contagem total de elétrons e é definido pela expressão:

$$
T E C=\int n_{e} d l
$$

O TEC é expresso em unidades de elétrons $/ \mathrm{m}^{2}$ ou ocasionalmente nas unidades do TEC (TECU), para $\boldsymbol{n}_{e}$ número de elétrons livres, sendo $1 \mathrm{TEC}=10^{16}$ elétrons livres $/ \mathrm{m}^{2}$. (Kaplan; Hegarty, 2006)

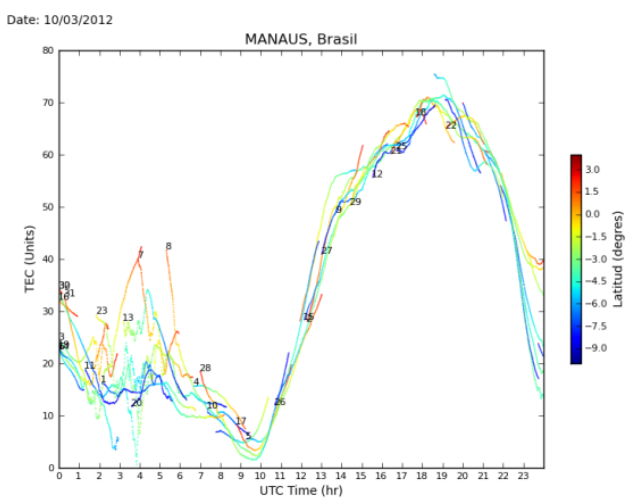

Figura 2 - Perfil de TEC no dia 10 de março de 2012 , sobre Manaus (Lat. -3,10 e Long. -59,97) mostrando durante a noite ocorrência de Bolhas de Plasma com forte deterioração do sinal GPS sobre o alvo entre 0 - 10 UTC. (Fonte: LISN - Low Latitude Ionospheric Sensor Network)

Para melhor compreensão do fenômeno estudado faz-se uso de uma ferramenta mais moderna no estudo das geociências, a transformada de ondeleta de Morlet. O fato básico sobre as ondeletas é que elas são localizadas no tempo (ou espaço), por isso denominada de funções de suporte compacto, contrariamente ao que ocorre com as funções trigonométricas. Por isso, tornaram-se ideais para analisar sinais não estacionários (BOLZAN, 2010). 
Um breve estudo de uma tempestade magnética e a formação de bolhas de plasma na região Amazônica, sobre Manaus (Lat. -3,10 e Long. -59,97)

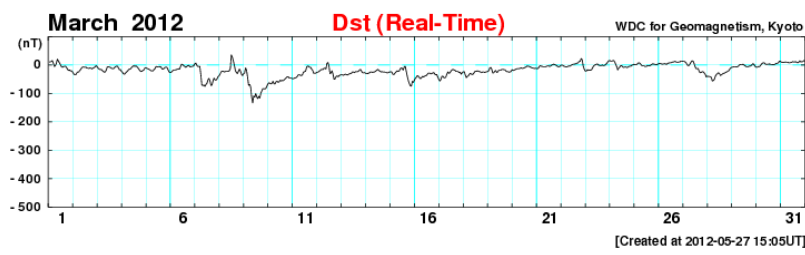

Figura 3. Série temporal de DST "Disturbance Storm Time", atenção para o dia 9 com DST de -133 nT. (Fonte: WDC for Geomagnetism, Kyoto).

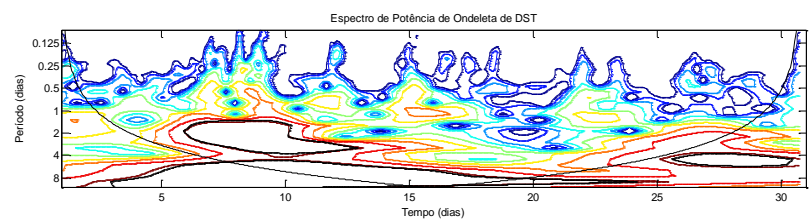

Figura 4. Observações diárias de Março de 2012 (Lat. 3,10; Long. -59,97), utilizando dados DST para a transformada de ondeleta de Morlet utilizando o pacote Torrence e Compo (1998).

Tabela 1 - Dados da Estação em Manaus - AM. (Fonte: UNIVAP - CEULM/ULBRA - LISN)

\begin{tabular}{|l|l|}
\hline Manaus & \\
\hline Country: & Brasil \\
\hline Network: & UNIVAP \\
\hline Latitude [degrees]: & -3.1082690 \\
\hline Longitude [degrees]: & -59.9746552 \\
\hline Altitude [meters]: & 45.4900017 \\
\hline Receiver: & NOV GSV4004B \\
\hline Antenna: & NOV702 rev.4 \\
\hline
\end{tabular}

\section{Resultados}

A Fig. 4 apresenta o gráfico em forma de ondeleta de Morlet. Neste é possível observarmos dentro do cone de influência a tempestade magnética de grande intensidade, ocorrida na dia 9 de março. Já nos dias 15 e 27 do mesmo mês, também ocorreram tempestades, porém com menos intensidade e baixa relevância. Quanto maior a degeneração do sinal GPS no perfil do TEC (Fig.2 e Fig. 5), este indicará tempestade magnética (Fig.3 e Fig.5) ou no período noturno também pode indicar a presença das bolhas de plasma (Fig. 2 entre $0-$ $5 \mathrm{~h} \mathrm{HL}$ ). Este fenômeno amplamente aceito como uma irregularidade gerada pela instabilidade do tipo RayleighTaylor associada a rápida subida da camada $F$ após o entardecer na região equatorial ocasionada pela reversão no campo elétrico do plasma ionosférico (Lima, 2009). O TEC pode atingir o seu máximo no período diurno e o seu mínimo no período noturno. Em Manaus a máxima pode ser atingida aproximadamente no horário das $14 \mathrm{~h}$. Enquanto a mínima ocorre entre $1 \mathrm{~h}$ às $5 \mathrm{~h}(\mathrm{HL})$.

\section{Discussão e Conclusões}

A transformada de ondeleta pode ser utilizada para o estudo de sinais não estacionários em diferentes frequências. A transformada de Morlet conserva energia não somente globalmente, mas também localmente ao se considerar todos os coeficientes dentro do cone de influência, o qual consiste no suporte espacial para toda a ondeleta (BOLZAN, 2010). Isto implica que não há perda de informação transformando 0 sinal em coeficientes de ondeleta (FARGE, 1996).

\section{Agradecimentos}

Aos orientadores Newton S. de Lima, Alan dos Santos Ferreira e Kaio Barbosa, também ao bolsista do projeto Matheus da Rocha Pietzsch pela ajuda computacional, todos do Centro Universitário Luterano de Manaus CEUM/ULBRA; a Fundação de Amparo e Pesquisa do Estado do Amazonas - FAPEAM pelos recursos; aos Doutores Paulo Roberto Fagundes, Y. Sahai, José R. Abalde, Valdir Gil Pillat da Universidade do Vale da Paraíba - UNIVAP/SJC-SP, pelo suporte da pesquisa; a FAPESP pelos equipqmentos da pesquisa; ao Dr. Maurício J. A. Bolzan da Universidade Federal de Goiás, pelo auxílio na aplicação da Transformada de Ondeleta. A LISN, através de http://lisn.igp.gob.pe/stations/status.php?page=tec\&statio $\mathrm{n}=$ man\#mapa. A Universidade de Kyoto através de http://wdc.kugi.kyoto-

u.ac.jp/dst_realtime/presenmonth/index.html.

\section{Referências}

Bolzan, M.J.A; Rosa, R.R - Transformada em Ondeletas: Teoria e Aplicações com Matlab. Goiana, 2010,

Burke, W. J.; Huang, C. Y.; Gentile, I. C.And Bauer, I., 2004. Seasonal-longitudinal variability of equatorial plasma bubbles. AnnalesGeophysicae 22, 3089-3098.

ECSS - E - 10-04 A. 2000 - Space engineering.Space environment (ECSS Secretariat. ESA - ESTEC, http://www.ecss.nl) acesso em janeiro 2007.

Farge, M.; Kevlahan N.; Perrier V.; Goirand E. Wavelets and turbulence. Proceedings ofthe IEEE, v. 84, n. 4, p. 639-669, Apr. 1996.

Kaplan, E. D.; Hegarty C. J.; Understanding GPS, Principles and Applications - Second Edition; Editora Artechhouse.

Lima, N. S. de, (Dissertação de Mestrado, 2009) UFAM

Torrence, C.; Compo, G. P. A Practical Guide to Wavelet Analysis. Bulletin of the American Meteorological Society, v. 79, n. 1, p. $61-78,1998$. 


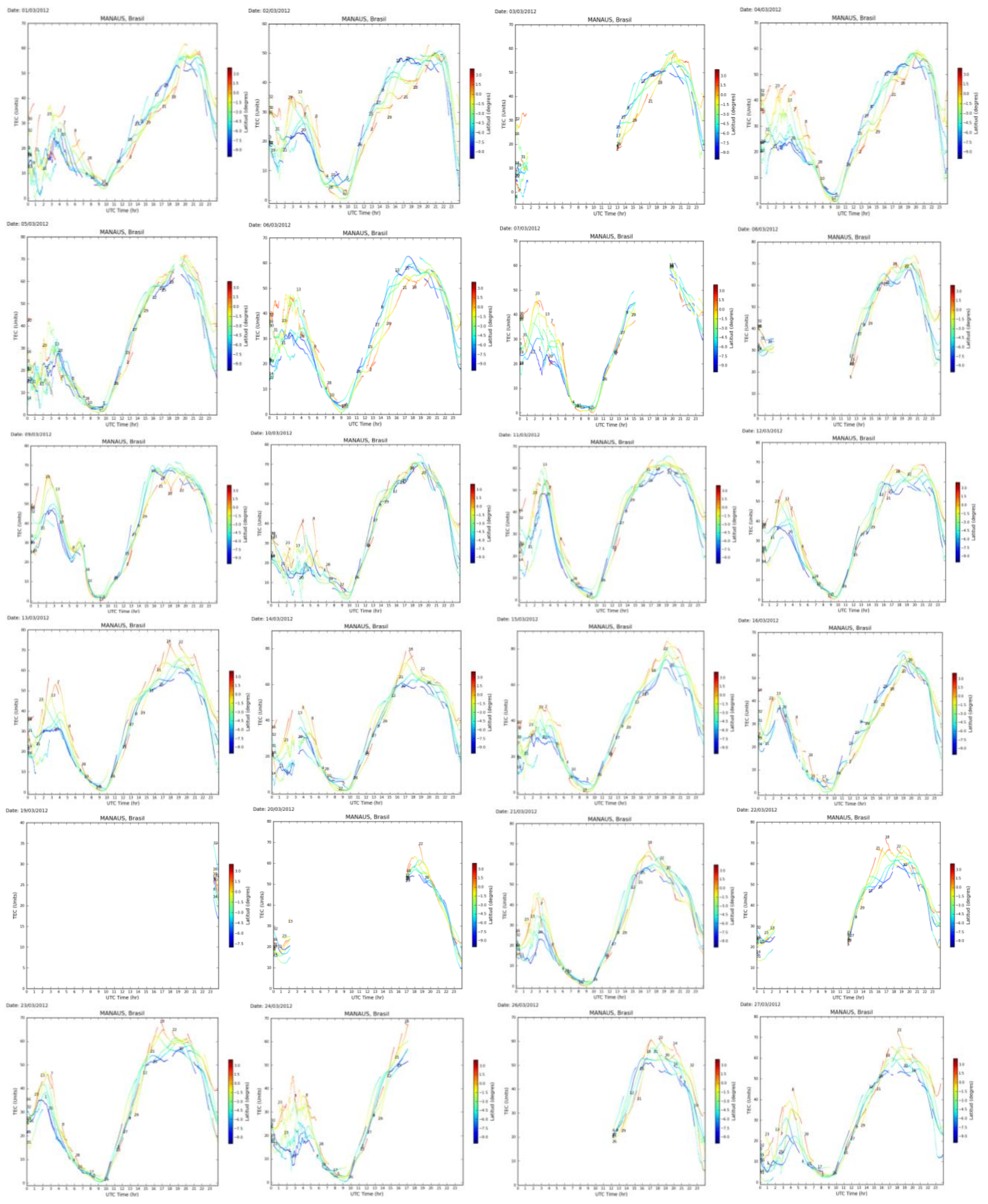

Figura 5: Perfis de TEC de Março de 2012 sobre Manaus, mostrando as tempestades magnéticas e as formações de bolhas de plasma na ionosfera equatorial no período noturno. (Fonte: http://lisn.igp.gob.pe/gps/) 